\section{A WORD FROM OLAW AND APHIS}

In response to the issues posed in this scenario, the National Institutes of HealthOffice of Laboratory Animal Welfare (NIH-OLAW) and the US Department of Agriculture-Animal and Plant Health Inspection Service (USDA-APHIS) provide the following clarifications:

In this scenario, the IACUC approved the renewal of a protocol involving survival surgery on a macaque without the analgesia regimen recommended by the veterinarian.

\section{NIH-OLAW response}

The IACUC's approval of the protocol is in direct conflict with the PHS Policy IV.C.1.b.and the Guide. The Policy explicitly requires the IACUC to determine that painful procedures will be performed with appropriate analgesia unless "justified for scientific reasons in writing by the investigator" did not obtain such a justification. The IACUC must also confirm that the protocol will be conducted in accordance with the USDA Animal Welfare Regulations which have similar requirements to the Policy as noted in the USDA response.

The Guide, page 121, states that "the selection of appropriate analgesics and anesthetics should reflect professional veterinary judgment as to which best meets clinical and humane requirements as well as the needs of the research protocol" ${ }^{2}$. The IACUC failed to rely on the veterinarian's professional judgement and instead opted for subjective observations from the principal investigator on the macaques' post-operative conditions.

Based on animal welfare concerns, the veterinarian's recourse is to request that the IACUC revisit review of the protocol. Obtaining outside consultation from others conducting similar procedures may improve the current dynamics. If the IACUC does not agree to amend the protocol, the veterinarian may file a minority view to document the concerns to the Institutional Official ${ }^{3}$. PHS Policy IV.F. requires institutions to include minority views in the annual report to OLAW $^{1,3}$. OLAW in turn would counsel the IACUC on their responsibility to ensure that animals receive adequate analgesia.

\section{USDA-APHIS response}

The Animal Welfare Act (AWA) regulations specify that the IACUC is to ensure procedures involving animals will avoid or minimize discomfort, distress, and pain to the animals ${ }^{4}$ and that the procedures that may cause more than momentary or slight pain or distress will be performed with appropriate sedatives, analgesics, or anesthetics, unless withholding such agents is justified for scientific reasons, in writing, by the principal investigator (PI) and will continue for only the necessary period of time ${ }^{5}$. The IACUC is also to ensure activities that involve surgery include the appropriate provision of pre-operative and post-operative care in accordance with established veterinary medical and nursing practices $^{6}$. The regulations require the PI to involve the Attending Veterinarian (AV) in the planning of an activity ${ }^{7}$ that will cause more than momentary and slight pain/distress. The AV is to provide the PI with guidance on handling, immobilization, anesthesia, analgesia, tranquilization, euthanasia, and adequate pre- and post-procedural care in accordance with current established veterinary medical and nursing procedures ${ }^{8}$. The research facility shall ensure that the AV has appropriate authority to ensure the provision of adequate veterinary care?

In this scenario, Boyd involved an $\mathrm{AV}$ (or designee) in the planning of the study. The veterinarian recommended Boyd include bupivacaine and meloxicam in the analgesic regimen to minimize pain and distress based on current veterinary practices and the fact this regimen was already in practice at the facility. Boyd however did not accept the veterinarian's recommendation because he believed buprenorphine post-operatively to be sufficient based on personal opinion. The study was approved after full Committee review.
The IACUC does not have the authority to prescribe methods or set standards of design, performance, or conduct of research ${ }^{10}$, but they have the authority, and the responsibility, to require modifications to secure approval or withhold approval ${ }^{11}$ of a proposal when procedures are not performed with adequate analgesics or anesthetic, or when no scientific justification for withholding analgesics is provided ${ }^{5}$. In this case Boyd did not provide a scientific justification for withholding the recommended analgesics. Noncompliances could result when approval of a protocol does not avoid or minimize pain or discomfort to the animals ${ }^{4}$ or when the veterinarian does not have the appropriate authority to ensure the provision of adequate veterinary care ${ }^{9}$, which includes adequate pre- and post-procedural care in accordance with current established veterinary medical and nursing procedures ${ }^{8}$.

Patricia Brown ${ }^{1 \star}$ and Betty Goldentyer ${ }^{2}$ ${ }^{1}$ Director, OLAW, OER, OD, NIH, HHS, Bethesda, United States. ${ }^{2}$ Acting Deputy Administrator, Animal Care, APHIS, USDA, Washington, USA. *e-mail:brownp@od.nih.gov

Published online: 18 December 2019 https://doi.org/10.1038/s41684-019-0446-Z

References

1. Office of Laboratory Animal Welfare, National Institutes of Health. Public Health Service Policy on Humane Care and Use of Laboratory Animals. (US Department of Health and Human Services, Bethesda, Maryland, USA, 2015).

2. Institute for Laboratory Animal Research. Guide for the Care and Use of Laboratory Animals. 8th ed. (National Academies Press, Washington D.C., 2011).

3. National Institutes of Health. Public Health Service Policy on Humane Care and Use of Laboratory Animals - Frequently Asked Questions. Institutional Reporting to OLAW, Question C.6. (US Department of Health and Human Services, Bethesda, MD, USA, revised 2017)

4. 9 C.F.R. $\$ 2.31(\mathrm{~d})(1)(\mathrm{i})$

9 C.F.R. $\$ 2.31$ (d)(1)(iv)(A)

6. 9 C.F.R. $\$ 2.31(\mathrm{~d})(1)(\mathrm{ix})$

7. 9 C.F.R. $\$ 2.31(\mathrm{~d})(1)(\mathrm{iv})(\mathrm{B})$

8. 9 C.F.R. $\$ 2.33(\mathrm{~b})(4)$

9. 9 C.F.R. $\$ 2.33(\mathrm{a})(2)$

10. 9 C.F.R. $\$ 2.31(\mathrm{a})$

11. 9 C.F.R. $\$ 2.31(c)(6)$

\title{
Embracing change
}

W e've all had to deal with change at some point in our lives and it takes time to acclimate to the way new things unfold. I'm sure that is the case for Boyd. He is comfortable, accustomed with the way his research has been running. When we talk about life forms, however, it is wise to embrace change.

Boyd is correct when he says that the veterinarian's role is to advise and consult, but the veterinarian is not limited to that. The veterinarian is responsible for the welfare of the animal and should exercise his/her professional judgement in that regard. In general animal practice, veterinarians' use only the most current medical and nursing procedureswhy not use these standards for our research animals? Analyzing pain is subjective, and as such a multi-modal approach to subside or even eliminate the pain is imperative.

I would have to say I am disappointed in the IACUC's decision. I agree, it is not by force that we change someone's opinion, but if we are not the animals' advocate, why are we even here?

\footnotetext{
Fatima Trujillo* and Laura Illingworth

California State Polytechnic University,

Pomona, CA, USA.

*e-mail:fjtrujillo27@gmail.com
}

Published online: 18 December 2019

https://doi.org/10.1038/s41684-019-0441-4 